Cahiers de la recherche sur les droits

Cahiers

Fur les Droits fondamentaux

$11 \mid 2013$

Le droit de la famille en (r)évolutions

\title{
La famille du majeur protégé : un environnement protecteur, destructeur ou victime
}

\section{Gilles Raoul-Cormeil}

\section{(2) OpenEdition}

\section{Journals}

Édition électronique

URL : https://journals.openedition.org/crdf/4655

DOI : $10.4000 /$ crdf.4655

ISSN : 2264-1246

Éditeur

Presses universitaires de Caen

\section{Édition imprimée}

Date de publication : 1 novembre 2013

Pagination : 63-75

ISSN : 1634-8842

\section{Référence électronique}

Gilles Raoul-Cormeil, « La famille du majeur protégé : un environnement protecteur, destructeur ou victime ", Cahiers de la recherche sur les droits fondamentaux [En ligne], 11 | 2013, mis en ligne le 01 décembre 2014, consulté le 15 novembre 2022. URL : http://journals.openedition.org/crdf/4655 ; DOI : https://doi.org/10.4000/crdf.4655 


\title{
La famille du majeur protégé: un environnement protecteur, destructeur ou victime
}

\author{
Gilles RAOUL-CORMEIL \\ Maître de conférences en droit privé à I'Université de Caen Basse-Normandie \\ Centre de recherches en droit privé (CRDP, EA 967)
}

I. Les familles appelées à protéger leurs majeurs vulnérables

A. La protection familiale officieuse

1. La subsidiarité contractuelle

2. La subsidiarité matrimoniale

B. La protection familiale officielle

1. L'ouverture de la mesure

2. Le fonctionnement de la mesure

II. Les familles écartées de la protection des majeurs vulnérables

A. Le recours au corps des mandataires judiciaires à la protection des majeurs (MJPM)

1. Les fondements de l'exclusion de la famille

2. Le statut de MJPM

B. Le rôle des MJPM dans l'exercice des droits familiaux

1. L'enfant

2. Le couple

Le livre premier du Code civil consacré aux personnes aura connu en l'espace de cinquante ans (1964-2013) une métamorphose ${ }^{1}$. Les effets paraissent toutefois inégaux selon qu'on se penche sur l'état civil ${ }^{2}$, le domicile, l'absence ${ }^{3}$, où la réécriture ne s'est heureusement guère départie de l'esprit de police civile qui les caractérise. Par contraste, la liberté individuelle et l'égalité ont insufflé le vent de la réforme sur le mariage 4 , le divorce 5 , l'autorité

1. Sur laquelle, voir P. Catala, «La métamorphose du droit de la famille», in Le Code civil: 1804-2004. Un passé, un présent, un avenir, Y. Lequette, L. Leveneur (dir.), Paris, Dalloz, 2004, p. 341-358; G. Cornu, Droit civil : la famille, $9^{\mathrm{e}}$ éd., Paris, Montchrestien (Domat droit privé), $2006, \mathrm{n}^{\circ} 6$ où l'auteur conclut à une "révolution tranquille». Voir aussi, parmi de nombreuses et riches études récentes, P. Malaurie, «La pensée juridique de Jean Carbonnier ", in Hommage à Jean Carbonnier (sous l'égide de l'association Henri Capitant), Paris, Dalloz (Thèmes et commentaires), 2007, p. 52-60; P. Catala, «L'esprit de la législation», in Hommage à Jean Carbonnier, p. 159-166. À comparer avec: F. Dekeuwer-Defossez, «L'accueil en législation", in Hommage à Jean Carbonnier, p. 207-220; Y. Lequette, "Quelques remarques sur le pluralisme en droit de la famille", in Mélanges en l'honneur du professeur Gérard Champenois, F. Bicheron et al. (dir.), Paris, Defrénois-Lextenso, 2012, p. 522-550.

2. Voir notamment la loi $\mathrm{n}^{\circ}$ 93-22 du 8 janvier 1993.

3. Loi no $77-1447$ du 28 décembre 1977.

4. Loi no $65-570$ du 13 juillet 1965 ; loi no $85-1372$ du 23 décembre 1985 , à propos des régimes matrimoniaux.

5. Loi no $75-617$ du 11 juillet 1975; loi no 2004-439 du 26 mai 2004 
parentale $^{6}$, l'adoption ${ }^{7}$, la filiation ${ }^{8}$, le nom ${ }^{9}$ mais aussi les successions ${ }^{10}$ et les libéralités. Le statut du corps humain ${ }^{11}$ et le pacte civil de solidarité (pacs) ${ }^{12}$ figurent enfin au titre des nouveautés. Signes de rupture, ces innovations se sont si bien installées dans le paysage juridique français que leur abrogation serait insensée. Ajoutées à l'architecture de l'édifice, celles-ci surchargent son sobre ordonnancement; mais dressées vers le ciel, ces flèches disciplinent les mœurs. Au titre des changements, la personne n'est plus ce masque abstrait, une signature qui contracte et répond de ses engagements patrimoniaux. La faveur qui lui a été accordée de fractionner le patrimoine en masses affectées à une activité professionnelle ${ }^{13}$ en modifie la définition. Mais l'unité se recentre sur cette volonté qui anime un corps humain, sans pouvoir disposer de ses éléments et produits comme s'ils étaient dans le commerce juridique de droit commun. Les liens entre les personnes sont plus que jamais pluriels. Le mariage n'est plus non plus le seul acte fondateur de la famille; il le reste néanmoins car les autres modes de conjugalité organisent la vie commune sans réunir les patrimoines ni unir les destinées jusqu'à la mort. Prenant ses distances avec le sacrement canonique puis avec les lois de la Nature, le droit du mariage a poursuivi son émancipation ${ }^{14}$. L'ordre

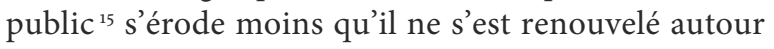
des valeurs qui nourrissaient autrefois ces institutions: la vertu de l'engagement, la prohibition de l'inceste ${ }^{16}$, la condamnation des violences intrafamiliales ${ }^{17}$ et la solidarité ont pris racine dans le droit civil. Modeste mais sûre, l'ambition de la fraternité est clairement affichée par le droit des aliments et le droit des personnes vulnérables.
Siège des règles qui organisent la vie sociale et pacifique des personnes, le Code civil n'est pas un droit d'exclusion. Sa rénovation se rattache désormais symboliquement à la devise républicaine: liberté, égalité fraternité.

Liberté, égalité, fraternité, une triade sous laquelle s'ordonnent aussi, plus précisément, les points de rencontre du nouveau droit des majeurs protégés avec la famille. La liberté d'abord, car la philosophie de la loi $\mathrm{n}^{\circ}$ 2007-308 du 5 mars 2007 est de protéger la personne sans la diminuer ${ }^{18}$, c'est-à-dire sans violer ses libertés individuelles, ses droits fondamentaux et finalement sa dignité ${ }^{19}$. Une liberté qui se présente comme la limite nécessaire à l'incapacité générale de la personne en tutelle qui a besoin d'être représentée pour les actes les plus importants de sa vie civile ${ }^{20}$. Même étendue au gouvernement de sa personne par-delà la gestion de ses biens, la tutelle se présente comme un sous-sol caverneux. L'autonomie du majeur protégé est l'image renversée de l'incapacité qui trouvait dans les silences de la loi du 3 janvier 1968 les voies de son développement ${ }^{21}$. Mais ce qui va sans dire va mieux en le disant. Aussi le législateur de 2007 a-t-il choisi de consacrer des droits strictement personnels qu'il a définis en contemplation «des actes dont la nature implique un consentement strictement personnel [et dont l'accomplissement] ne peut jamais donner lieu à assistance ou à représentation de la personne protégée ${ }^{22}$. Consacrée sur l'autel du droit naturel, cette capacité minimale et irréductible se concentre sur la postérité du majeur protégé, le fruit de sa vie intime. En revanche, l'union, comme la désunion des liens de couple, la santé et la relation médicale ${ }^{23}$ ne peuvent être

6. Loi $\mathrm{n}^{\circ} 70-459$ du 4 juin 1970 ; loi $\mathrm{n}^{\circ}$ 87-570 du 22 juillet 1987 ; loi $\mathrm{n}^{\circ} 2002-305$ du 4 mars 2002.

7. Loi $n^{\circ} 66-500$ du 11 juillet 1966 ; loi $n^{\circ} 76-1179$ du 22 décembre 1976.

8. Loi $\mathrm{n}^{\circ} 72-5 \mathrm{du} 3$ janvier 1972 ; ordonnance $\mathrm{n}^{\circ} 2005-759 \mathrm{du} 4$ juillet 2005, ratifiée par la loi no $2009-61$ du 16 janvier 2009.

9. Loi no 2002-304 du 4 mars 2002, modifiée par la loi no $2003-516$ du 18 juin 2003.

10. Loi $\mathrm{n}^{\circ}$ 2001-1135 du 3 décembre 2001; loi no $2006-728$ du 23 juin 2006.

11. Loi $n^{\circ} 94-653$ du 29 juillet 1994

12. Loi $n^{\circ} 99-944$ du 15 novembre 1999 ; loi no $2006-728$ du 23 juin 2006.

13. Loi n 2010-658 du 15 juin 2010 sur l'entrepreneur individuel à responsabilité limitée.

14. Loi $\mathrm{n}^{\circ}$ 2013-404 du 17 mai 2013 ouvrant le mariage aux couples du même sexe (art. $1^{\mathrm{er}}$ ). Voir aussi CC, déc. nº $2013-669$ DC du 17 mai 2013, JORF, 18 mai 2013, p. 8281 .

15. Sur lequel, voir spécialement J. Hauser, «L'ordre public et le droit familial - Rapport français », in L'ordre public. Travaux de l'association Henri Capitant et des amis de la culture juridique, t. XLIX, Journées libanaises, Paris, LGDJ, 1998, p. 475-492.

16. Voir A. Batteur, «L'interdit de l'inceste, principe fondateur du droit de la famille», Revue trimestrielle de droit civil, 200o, p. 759-780.

17. Loi $\mathrm{n}^{\circ}$ 2010-769 du 9 juillet 2010.

18. Voir T. Fossier, «L'objectif de la réforme du droit des incapacités: protéger sans jamais diminuer», Defrénois, 2005, art. 38076, p. 3-34. Voir aussi P. Malaurie, «La réforme de la protection juridique des majeurs (loi n 2007-308 du 5 mars 2007)», Defrénois, 2007, art. 38569, p. 557-572; J. Hauser, "Des incapables aux personnes vulnérables», Droit de la famille, mai 2007, étude ${ }^{\circ}{ }_{14}$; T. Fossier, "La réforme de la protection des majeurs. Guide de lecture de la loi du 5 mars 2007", La semaine juridique, éd. G, 2007, I, 118, p. 13-23; Revue trimestrielle de droit civil, 2007, p. 394-407, obs. A.-M. Leroyer.

19. Code civil, art. 415, al. 2.

20. Code civil, art. 440, al. 3 .

21. Voir spécialement J. Hauser, «Réflexions sur la protection de la personne de l'incapable», in Mélanges offerts à Pierre Raynaud, Paris, Dalloz-Sirey, 1985, p. 227-236; T. Fossier, «Le gouvernement de la personne incapable majeure», La semaine juridique, éd. G, 1985, I, 3195; J.-P. Gridel, «L'acte éminemment personnel et la volonté propre au majeur en tutelle», Rapport de la Cour de cassation, 200o, p. 79-92.

22. Code civil, art. 458 , al. $\mathbf{1}^{\text {er }}$. Ce texte a donné lieu à de nombreuses études: T. Fossier, «Le statut civil de la personne vulnérable, gouverné par des principes fondamentaux», La semaine juridique, éd. N, 2008, 1277; A. Batteur, "Majeurs protégés. Curatelle et tutelle. Effets personnels. Articles 457-1 à 463", Jurisclasseur Code civil, fascicule n 31, 2008, passim; D. Guével, «La protection des actes personnels et familiaux des majeurs vulnérables», Les petites affiches, n 220, 4 novembre 2010, p. 30-34; J. Hauser, "Une théorie générale de la protection du sujet vulnérable?», Revue Lamy droit civil, $\mathrm{n}^{\circ}$ 83, juin 2011, étude n 4288, p. 69-73; S. Moracchini-Zeidenberg, "L'acte personnel de la personne vulnérable», Revue trimestrielle de droit civil, 2012, p. 21-32.

23. Voir spécialement F. Arhab-Girardin, «La décision médicale du majeur protégé: une articulation complexe des dispositions du code de la santé publique avec la loi du 5 mars 2007 portant réforme de la protection juridique des majeurs », Revue de droit sanitaire et social, 2009, p. 875-889; 
mis en œuvre sans l'intervention de la personne chargée de la mesure de protection au risque d'exposer l'intéressé à un dénuement certain. Le majeur protégé est donc aussi protégé dans l'exercice de ses droits familiaux.

Égalité ensuite, la loi du 5 mars 2007 regarde toutes les formes de vie de couple avec la même confiance. Peu importe la nature des liens de fait (concubinage) ou de droit (mariage et pacs) unissant le majeur protégé à la personne qui partage sa vie, la loi la place en bonne situation pour saisir le juge des tutelles et solliciter d'être désignée pour exercer la charge curatélaire ou tutélaire. Toutefois l'égalité affermie par le nouveau droit des majeurs protégés n'est pas absolue. La loi du 5 mars 2007 n'avait pas pour objet de combler les lacunes du droit du pacs en contemplation du droit des régimes matrimoniaux.

Fraternité enfin, la loi du 5 mars 2007 moralise les rapports familiaux: la protection d'un majeur vulnérable dans sa personne et ses biens "est un devoir des familles» ${ }^{24}$. La proclamation d'un tel devoir témoigne du besoin de l'État de se reposer sur les solidarités familiales, évitant ainsi de poser l'équation «grand âge $=$ mise sous tutelle ${ }^{25}$. Le principe de subsidiarité et le principe de préférence familiale ne sont cependant pas non plus absolus. L'intérêt du majeur protégé peut justifier d'écarter la famille dans la conduite de la curatelle ou de la tutelle, bien que la loi abandonne à l'appréciation des juges du fond le soin de fixer le critère de désignation d'un professionnel. Si certaines maladies mentales trouvent dans un climat familial confus et oppressant les ressources de leur aggravation, les proches des majeurs protégés revêtent aussi bien les habits de bourreau que de victime. Les livres de jurisprudence sont peu bavards sur ces dérives familiales qui justifient une intervention des services sociaux sous le contrôle judiciaire.

Réaliste, la loi du 5 mars 2007 regarde les familles dans les yeux, avec leurs qualités et leurs défauts. Aux familles bienfaisantes, l'État est reconnaissant. Gare aux autres, l'État est menaçant. Sans ambiguïté ${ }^{26}$, mais avec ambivalence et nuances, la loi du 5 mars 2007 fait donc la part belle aux familles, et spécialement aux familles mariées, lorsqu'elle appelle les époux par-delà les parents à exercer la charge curatélaire ou tutélaire (I). En remède aux conflits et désordres de toute nature, la loi permet cependant au juge d'écarter les membres de la famille de la dévolution de la magistrature curatélaire ou tutélaire (II).

\section{Les familles appelées à protéger leurs majeurs vulnérables}

La loi étire largement le diamètre du cercle familial lorsqu'elle appelle ses membres à jouer un rôle dans la protection des personnes vulnérables, c'est là une différence assez nette avec le cercle des obligés alimentaires ${ }^{27}$ ou celui de la famille mortis causa limité aux parents unis au défunt par le lien du mariage ou de la parenté collatérale au sixième degré ${ }^{28}$. Le rôle dévolu à la famille est plus ou moins ouvert, plus ou moins étendu aussi selon le fondement retenu: le mariage ou le contrat. Dans tous les cas, le juge des tutelles a le dernier mot en cas de difficultés pour substituer à la protection familiale officieuse (A) une protection familiale officielle (B).

\section{A. La protection familiale officieuse}

Tout le droit des majeurs protégés est construit sur le principe de nécessité ${ }^{29}$. Nécessité médicale: le juge ne peut pas être régulièrement saisi si la requête n'est pas accompagnée d'un certificat médical circonstancié qui constate l'altération des facultés mentales du sujet et son besoin d'être assisté ou représenté dans les actes importants de la vie courante. Nécessité judiciaire: seul le juge, gardien des libertés individuelles, peut diminuer la pleine capacité civile d'une personne majeure, puisque cette décision est aussi restrictive de la liberté individuelle. Nécessité individuelle: le sujet vulnérable peut avoir anticipé sur sa faiblesse à venir. Au mandat de protection future et aux diverses procurations signées, le mariage est une alternative qui rend inutile l'ouverture d'une mesure de protection juridique. La subsidiarité est un corollaire du principe de nécessité sur le fondement de laquelle une protection officieuse peut être organisée. Seul le mandat de protection future offre au mandant une liberté intuitu personae puisqu'il n'est pas contraint de choisir le mandataire parmi les membres de la famille (1), contrairement au mariage qui désigne l'élu (2).

A. Batteur, «Recherche d'une articulation entre le Code de la santé publique et le Code civil: un défi à relever en faveur des personnes vulnérables», Droit de la famille, février 2011, étude n 5 , p. 22-27; G. Raoul-Cormeil, «Remèdes à l'éclatement du régime juridique des actes médicaux portant sur le majeur protégé», in Nouveau droit des majeurs protégés: difficultés pratiques, G. Raoul-Cormeil (dir.), Paris, Dalloz, 2012, p. 151-172.

24. Code civil, art. 415, al. 3. Cette directive est étendue à la protection des mineurs orphelins ou dont les père et mère se trouvent privés de l'exercice de l'autorité parentale; voir Code civil, art. 494.

25. J. Favard, «La protection des personnes âgées vulnérables», in Droit et vieillissement de la personne, J.-R. Binet (dir.), Paris, LexisNexis-Litec (Colloque et débats), 2008, p. 8.

26. Voir N. Peterka, «La famille dans la réforme de la protection juridique des majeurs», La semaine juridique, éd. G, 2010, étude nº 33 , où l'auteur distingue la famille à l'honneur et la famille à l'épreuve.

27. Code civil, art. 203 à 211.

28. Code civil, art. 734 et 743 .

29. Voir M. Rebourg, "Les principes directeurs de la loi du 5 mars 2007 réformant la protection juridique des majeurs ", Droit de la famille, mai 2007 , étude $n^{\circ}$ 16, p. 11-14; J.-M. Plazy, «Majeurs protégés (mars 2006-juin 2007) », Dalloz, 2008, Panor., p. 313: «Nécessité, subsidiarité et proportionnalité». Cette trilogie a été enrichie par un auteur: T. Fossier, Curatelle, tutelle, accompagnements: protection des mineurs et des majeurs vulnérables, T. Fossier (dir.), Paris, LexisNexis-Litec, 2009, nº 12 sq., p. 8-16 («Nécessité, subsidiarité, proportionnalité, dignité, solidarité et responsabilité»). 


\section{La subsidiarité contractuelle}

Inspiré du mandat d'inaptitude future ${ }^{30}$ en pratique au Québec, le mandat de protection future est la réponse du législateur à l'angoisse des personnes qui redoutent d'être une charge pour les membres de leur famille. La loi de 2007 leur permet d'anticiper sur leur fin de vie ${ }^{31}$ et d'organiser avec une personne de confiance la gestion de leurs biens et la protection de leur personne. Le mandat de protection future est assez facile à conclure. Il suffit aux parties de remplir le modèle réglementaire ${ }^{32}$. Si elles veulent s'en écarter, elles devront solliciter le ministère d'un avocat ${ }^{33}$. Mais ce mandat sous seing privé n'offre pas plus d'avantages qu'une procuration ${ }^{34}$ car le pouvoir du mandataire se réduit à l'accomplissement d'actes d'administration ${ }^{35}$, ce qui ne fait que repousser le moment où il deviendra nécessaire de saisir le juge des tutelles pour ouvrir une mesure judiciaire de protection. Plus ambitieux est le mandat notarié qui investit le mandant de «tous les actes patrimoniaux que le tuteur peut accomplir seul ou avec une autorisation ${ }^{36}$. La loi a élevé des garde-fous à cette large investiture du mandant qui embrasse les actes d'administration et de disposition. D'abord, le mandataire ne pourra pas exercer son pouvoir de représentation sans avoir préalablement fait constater l'altération des facultés mentales par un médecin choisi sur la liste du procureur de la République ${ }^{37}$, fait viser le mandat par le greffier du tribunal d'instance et fait notifier sa prise d'effet au mandant pour le cas où celui-ci ne pourrait pas l'accompagner lors de sa prise d'effet ${ }^{38}$. Ensuite, le mandataire devra établir un inventaire et rendre compte au notaire chaque année de l'exercice de sa mission, à charge pour le notaire d'alerter le juge des tutelles de la gestion hasardeuse du mandataire qui seul pourra mettre fin au mandat ${ }^{39}$. Enfin, le mandataire devra solliciter exceptionnellement une autorisation du juge des tutelles pour la conclusion des actes à titre gratuit ${ }^{40}$, mais également pour disposer des immeubles affectés aux résidences du mandant qui a perdu raison. L'application de l'article $426 \mathrm{du}$ Code civil, implicitement souhaitée par le législateur qui a placé ce texte dans les dispositions générales et communes à toutes les mesures de protection juridique, y compris au mandat de protection future, aurait vidé cette innovation légale d'une bonne part de son intérêt. L'infortune du mandat de protection future est cependant ailleurs. Puisque la capacité est d'ordre public et partant indisponible, l'activation du mandat de protection future laisse au mandant insane sa pleine capacité civile ${ }^{41}$. Le notariat propose d'y remédier partiellement en inscrivant les mandats notariés sur le fichier central des dispositions de dernière volonté ${ }^{42}$ afin d'avertir le notaire de ne pas laisser conclure un acte qui pourrait être annulé sur le fondement de l'article 414-1 du Code civil par le mandataire ou les héritiers du mandant. La publicité de ce mandat permettrait également de prévenir le risque qu'un mandant ne dispose d'un immeuble concurremment avec son mandataire, ce qui créerait le risque d'une aliénation successive d'un même bien par la même personne, ici présente et là représentée. Il reste à espérer que les familles trouveront avec les praticiens les moyens de rédiger des contrats qui satisferont les intérêts du mandant car les premières manifestations jurisprudentielles ont plutôt montré les efforts déployés avec hâte par certains parents de personnes âgées pour priver les juges des tutelles de tout pouvoir d'investigation ${ }^{43}$ et d'action ${ }^{44}$. Le mariage est une alternative au dispositif tutélaire.

30. Sur les origines de cette innovation, voir D. Noguéro, «Interrogations au sujet du mandat de protection future», Dalloz, 2006, Chron., p. 1133-1138.

31. De ce point de vue, le mandat de protection future peut être rapproché des directives anticipées. Sur ces liens, voir J. Hauser, «Les mesures judiciaires, solution subsidiaire au mandat de protection future? », in Nouveau droit des majeurs protégés..., p. 13-24, passim.

32. Voir le décret no 2007-1702 du 30 novembre 2007, modifié par le décret n²009-1628 du 23 décembre 2009, comportant en annexe un modèle et une notice d'information.

33. Code civil, art. 492.

34. Voir L. Leveneur, "Intérêts et limite du mandat de protection future», in Mélanges en l'honneur du professeur Gérard Champenois, p. 575 in fine: «On finit par ne plus trop discerner ce à quoi peut s'étendre le mandat de protection future [...] Si bien que la différence avec un mandat ordinaire n'est pas très sensible».

35. Code civil, art. 493 .

36. Code civil, art. 490 , al. $1^{\text {er }}$.

37. Code civil, art. 481, al. 2 .

38. Code de procédure civile, art. 1258-4 (Rédac. décret nº 2008-1276 du 5 décembre 2008)

39. Code civil, art. $483,4^{\circ}$.

40. Code civil, art. 490, al. 2

41. Voir, sur ce point, J. Klein, «Le mandat de protection future ou la protection juridique conventionnelle», Droit de la famille, mai 2007, étude $\mathrm{n}^{\circ}$ 21, p. 36-38; A. Batteur, "Contrat et mesures de protection», La semaine juridique, éd. N, 2008, 1275; D. Fenouillet, «Le mandat de protection future ou la double illusion ", Defrénois, 2009, art. 38882, p. 142-177; P. Malaurie, "Le mandat en droit des personnes", in Le mandat, un contrat en crise?, N. Dissaux (dir.), Paris, Economica (Études juridiques; 37), 2011, p. 115-125; L. Leveneur, «Intérêts et limite du mandat... », p. 576.

42. Sur cette proposition et bien d'autres, voir D. Noguéro, "La publicité des mesures de protection des majeurs", in Mélanges en l'honneur du professeur Jean Hauser, P. Delmas Saint-Hilaire, A. Gouttenoire, M. Lamarche et al. (dir.), Paris, LexisNexis - Dalloz, 2012, p. 467-533, spéc. p. 526; Revue trimestrielle de droit civil, 2013, p. 87, obs. J. Hauser.

43. Voir spécialement Cass., $1^{\mathrm{re}}$ civ., 12 janvier 2011, no 09-16.519, L'actualité juridique. Famille, 2011, p. 110, note T. Verheyde; Dalloz, 2011, Jur., p. 1204, note D. Noguéro; Droit de la famille, mars 2011, comm. n 42, note I. Maria; La semaine juridique, éd. G, 2011, 416, note N. Peterka; La semaine juridique, éd. $N$, 2011, 1115, note D. Boulanger, et 1244, chron. J. Massip; L'essentiel droit de la famille et des personnes, mars 2011, comm. ${ }^{\circ}$ 035, obs. G. Raoul-Cormeil; Revue juridique personnes et famille, 2011-4/12, note A. Caron-Déglise; Revue trimestrielle de droit civil, 2011, p. 324, obs. J. Hauser. Voir aussi Cass. $1^{\text {re }}$ civ., 29 mai 2013, n 12-19.851, Dalloz, 2013, Jur., p. 1815, note D. Noguéro.

44. Voir spécialement J. Massip, «De quelques problèmes posés par la protection juridique des majeurs», La semaine juridique, éd. N, 2011, 1244; H. Fulchiron, "Peut-on protéger la personne contre elle-même? Réflexions autour de l'affaire Bettencourt», in Mélanges en l'honneur de la professeure Françoise Dekeuwer-Defossez, Michel Dupuis (dir.), Paris, Montchrestien-Lextenso, 2012, p. 117-145. Voir aussi, du même auteur: 


\section{La subsidiarité matrimoniale}

L'article $428 \mathrm{du}$ Code civil fait ordre au juge de ne pas ouvrir de mesure de protection juridique s'il peut être pourvu aux intérêts d'une personne souffrant d'une altération de ses facultés mentales par l'application du droit du mariage, des droits et devoirs respectifs entre époux ainsi que des règles des régimes matrimoniaux. La prééminence du mariage sur le dispositif tutélaire a été organisée par la loi du 3 janvier 1968, même si l'ancien article $498 \mathrm{du}$ Code civil n'a fait que développer une institution qui est née pendant les années obscures de l'occupation nazie où les pouvoirs publics avaient organisé le service du travail obligatoire. C'est une loi du 22 septembre 1942 qui a enrichi l'article $213 \mathrm{du}$ Code civil:

La femme remplace le mari dans sa fonction de chef de famille s'il est hors d'état de manifester sa volonté en raison de son incapacité, de son absence, de son éloignement ou de toute autre cause ${ }^{45}$.

Validé par l'ordonnance du 9 octobre 1945, ce texte a permis à une femme mariée d'exercer les fonctions de chef de famille si son mari souffrait d'une «aliénation mentale ${ }^{46}$. Or,

Si l'on va au fond des choses [écrivait le professeur Carbonnier, en 1947], ce n'est pas dans la personne dont la manifestation de volonté est attendue qu'il convient de placer le centre de gravité de la notion légale [de mesure de crise], mais bien dans la notion de personne qui attend cette manifestation de volonté. L'impossibilité de manifester sa volonté $[\ldots]$ n'a pas été prise en considération par la loi [c'est-à-dire le droit du mariage] pour la protection de celui qui ne peut agir. C'est, en réalité, une organisation particulière de la théorie de la force majeure ou, si l'on veut, de la théorie de la nécessité, dans l'intérêt de ceux qui ont des droits subordonnés à la volition d'autrui. Devant l'impossibilité d'obtenir cette volition indispensable, comme devant n'importe quelle nécessité, le droit doit fléchir les règles ordinaires, et le consentement qui manque sera suppléé ou supposé ${ }^{47}$.

En qualité d'auteur de l'avant-projet réformant le droit des majeurs incapables, le doyen Carbonnier ajoutait au Code civil la règle selon laquelle [i]l n'y a pas lieu d'ouvrir une tutelle qui devrait être dévolue au conjoint, si, par application du régime matrimonial [...] il peut être suffisamment pourvu aux intérêts de la personne protégée ${ }^{48}$.

Les remèdes portés par le droit des régimes matrimoniaux furent cependant aussi nombreux qu'inefficaces. Il restait bien plus simple à l'époux diligent de signaler au juge des tutelles l'embarras dans lequel il était placé plutôt que de saisir, par la grâce du ministère d'un avocat, le tribunal de grande instance par une requête tendant à l'investir du pouvoir de passer seul un acte juridique qui requerrait le consentement des deux époux ou celui du conjoint propriétaire aujourd'hui empêché de manifester sa volonté. Un rapport qui fit grand bruit à la Chancellerie rappela aux juges des tutelles l'impérieux besoin d'observer le principe de subsidiarité ${ }^{49}$, mais il a fallu encore attendre le décret d'application de la loi réformant le divorce, en 2004, pour que le gouvernement modifiât le Code de procédure civile et facilitât la tâche des époux diligents ${ }^{50}$. Compétent pour ouvrir une mesure de protection juridique, le juge des tutelles est à même de faire respecter le principe de subsidiarité puisqu'il lui suffit d'autoriser son conjoint à représenter l'époux insane (ou privé de la faculté de s'exprimer) pour administrer un bien ou conclure un acte de disposition. S'il s'agit de vendre l'immeuble affecté à l'habitation du couple marié ${ }^{51}$, l'époux diligent signera donc deux fois l'instrumentum: une fois pour lui-même et une autre fois pour son conjoint s'il est habilité à le représenter pour cet acte. L'article 219 du Code civil a une portée ponctuelle mais assez large puisque ce texte du statut impératif de base ignore la distinction entre les régimes séparatistes et communautaristes ${ }^{52}$. En revanche, le juge aux affaires familiales est devenu par un décret de 2009 le seul juge compétent pour habiliter un époux à passer seul un acte qui requerrait le consentement des deux époux si le conjoint ne s'y opposait par entêtement en foulant au pied l'intérêt de la famille ${ }^{53}$. C'est ce juge spécialisé du tribunal de grande instance qui est seul compétent pour interdire au conjoint tourmenté de passer seul des actes juridiques sur ses propres biens afin de prévenir le risque de mise en péril des intérêts de la famille ${ }^{54}$, en dépensant sans compter par exemple. Si l'application des articles 217, 220-1 à 220-3,

«Peut-on protéger la personne contre elle-même?», La semaine juridique, éd. N, 2012, 1196; E. Paillet, «L'opposition de la personne majeure vulnérable à sa protection", in Mélanges en l'honneur du professeur Jean Hauser, p. 535-548.

45. Sur cet article, voir H. Solus, "Mari et femme selon la loi du 22 septembre 1942 », Revue trimestrielle de droit civil, 1943, p. 81-98; L. Julliot de la Morandière, "La loi du 22 septembre 1942 sur les effets du mariage quant aux droits et devoirs des époux", La semaine juridique, 1943, I, 304; M. de Juglart, «Les pouvoirs de la femme commune en biens depuis la loi du 22 septembre 1942», La semaine juridique, 1943, I, 327.

46. CA Poitiers, Dame Cottet, 13 novembre 1946, Dalloz, 1947, Jur., 2, p. 397-400, note J. Carbonnier («L'habilitation de la femme à représenter son mari aliéné...»).

47. Dalloz, 1947, Jur., 2, note J. Carbonnier, p. 398 sq.

48. Code civil, anc. art. 498 (Rédac. loi nº 68-5 du 3 janvier 1968). Rappr. Code civil, art. 428 (Rédac. loi n 2007-308 du 5 mars 2007).

49. F. Delbano, «Les difficultés d’application des principes de nécessité et de subsidiarité des régimes de protection des majeurs ", Dalloz, 1999, Chron., p. 408-413, spéc. p. 411.

50. Code de procédure civile, art. 1286, al. 2 (Rédac. décret nº 2004-1158 du 29 octobre 2004).

51. Code civil, art. 215, al. 3. La vente du logement et de ses meubles le garnissant est soumise à gestion conjointe que ces biens soient propres, communs ou indivis.

52. Les communautés font l'objet de remèdes plus appropriés, régis par les articles 1426 et 1429 du Code civil.

53. Code civil, art. 217 .

54. Code civil, art. 220-1 à 220-3. 
1426 et 1429 prime le droit des majeurs protégés, on ne peut s'empêcher de les juger complexes. Si peu propices au maintien de la vie conjugale, ne sont-ils pas plutôt le marchepied de la procédure de divorce ${ }^{55}$ ? Ces mécanismes demeurent néanmoins l'apanage du droit du mariage. Si les partenaires liés par un pacs sont aussi soumis à un devoir d'assistance ${ }^{56}$, seuls les époux sont mariés pour le meilleur et pour le pire, et investis, dans le cas où l'un d'eux sombrerait dans la folie, de prérogatives exorbitantes du droit commun analogues à un changement de régime matrimonial ${ }^{57}$. Ceci étant dit, l'époux habilité à gérer seul les biens propres de son conjoint ou la communauté n'a pas plus de pouvoirs qu'un tuteur. Alors que ce dernier doit solliciter le juge des tutelles pour conclure chaque acte de disposition ${ }^{58}$, l'époux commun en biens devra être autorisé par le juge aux affaires familiales pour conclure un acte soumis à cogestion ${ }^{59}$, ce qui revient - vérification faite - au même ${ }^{60}$. Les remèdes aux crises matrimoniales présentent l'avantage de constituer une protection discrète, sans émargement de la mention « $\mathrm{RC}{ }^{61}$ sur l'acte de naissance de l'époux souffrant d'une altération de ses facultés mentales. L'époux empêché conserve ainsi sa pleine capacité civile jusqu'à ce que son conjoint décide de rendre officielle cette vulnérabilité.

\section{B. La protection familiale officielle}

La famille très largement conçue peut jouer un rôle décisif dans l'ouverture (1) et le fonctionnement de la mesure de protection juridique (2).

\section{L'ouverture de la mesure}

La famille est au cœur de l'entourage de la personne vulnérable sans avoir de titre exclusif. Révérence faite à l'intéressé auquel la loi permet de solliciter du juge des tutelles l'ouverture d'une mesure de protection, l'article $430 \mathrm{du}$ Code civil regarde ensuite le couple. Traitant à égalité les modes de conjugalité (mariage, pacs), la loi exige du requérant la démonstration d'un lien réel plutôt que formel avec la personne à protéger. La vie commune ne doit pas avoir cessé entre eux (concubins, partenaires liés par un pacs), ce qui ne signifie pas qu'une séparation de toit soit synonyme de désintérêt pour la personne souffrante avec laquelle elle reste mariée ou pacsée. Si les séparations de fait sont suspectes, l'hospitalisation d'une personne illustre mieux le cas où des personnes vivent séparées sans avoir rompu leur communauté de vie. L'article $430 \mathrm{du}$ Code civil ne s'arrête pas au couple. La famille s'ouvre alors sur les parents et les alliés, cependant visés au singulier par ce texte qui habilite un requérant. Les parents sont les ascendants et les descendants en ligne directe, mais aussi les membres de la famille adoptive ou par le sang qui ont un auteur commun ${ }^{62}$. Les collatéraux privilégiés sont les frères et sœurs. Les oncles et tantes, neveux et nièces, cousins germains, utérins ou consanguins entrent dans le grand cercle des collatéraux ordinaires. Quant aux alliés, il s'agit des parents du conjoint: la famille par alliance embrasse les père et mère du conjoint, ses frères et sœurs et leurs enfants. L'article $430 \mathrm{du}$ Code civil ne s'arrête pas aux liens du sang et de l'alliance, elle accorde sa confiance à toute personne qui entretient avec l'intéressé des liens étroits et stables. L'ami, doublé d'un voisin, n'est donc pas sans titre.

Aucun d'eux toutefois ne pourra valablement saisir le juge des tutelles si sa requête n'est pas accompagnée d'un certificat médical circonstancié. Inviter une personne vulnérable à se laisser examiner par un médecin choisi sur la liste du procureur de la République n'est pas chose aisée lorsque la demande est faite par un enfant qui éprouve le sentiment de porter atteinte à l'honneur ou au respect de son père ou de sa mère. Réciproquement, bien des personnes sont en déni de leur maladie mentale parce qu'elles n'en sont qu'au début de leur développement et que celle-ci ne les prive pas de continuer à exercer leurs affaires. Naguère, la Cour de cassation avait jugé que la personne protégée «n'est pas fondée à se prévaloir de l'absence de constatation médicale de l'altération de ses facultés lorsque, par son propre fait, elle a rendu cette constatation impossible en se refusant à tout examen ${ }^{63}$. Si la consultation des travaux préparatoires ${ }^{64}$ de la loi du

55. Sur cette idée, voir G. Raoul-Cormeil: «Le logement de la famille et l'article 217 du Code civil (à propos de Cass., $1^{\text {re }}$ civ., 30 septembre 2009 , no 08-13.220)", Gazette du palais, no 314-315, 10-11 novembre 2010, p. 17-22.

56. Code civil, art. 515-4, al. $1^{\mathrm{er}}$ : «Les partenaires liés par un pacte civil de solidarité s'engagent à une vie commune, ainsi qu'à une aide matérielle et une assistance réciproques".

57. Code civil, art. 1396, al. 3: «Le mariage célébré, il ne peut être apporté de changement au régime matrimonial que par l'effet d'un jugement à la demande de l'un des époux dans le cas de la séparation de biens ou des autres mesures judiciaires de protection [...] ».

58. Code civil, art. 1426, al. 2 : «Le conjoint ainsi habilité par justice a les mêmes pouvoirs qu'aurait eux l'époux qu'il remplace; il passe avec l'autorisation de justice les actes pour lesquels son consentement aurait été requis s'il n'y avait pas eu substitution».

59. Voir notamment les articles 1422,1424 et 1425 du Code civil.

60. Pour une démonstration, voir Cass., $1^{\text {re }}$ civ., $1^{\text {er }}$ février 2012, nº 11-11.346, Dalloz, 2012, Jur., p. 921, note G. Raoul-Cormeil («La subsidiarité du dispositif tutélaire face au mariage avec communauté universelle»). Voir aussi Droit de la famille, mars 2012, comm. $\mathrm{n}^{\circ} 53$, note I. Maria; La semaine juridique, éd. N, 2012, 1183, note J. Massip. Dans cet arrêt de rejet, la Cour de cassation souligne que «les actes qui [...] étaient reprochés [à l'épouse de la personne vulnérable par leur fils] n'établissaient pas un risque de dilapidation des biens communs».

61. Code de procédure civile, art. 1233, al. ${ }^{\text {er }}$, qui prévoit qu'un extrait de toute décision portant ouverture, modification ou mainlevée de la curatelle ou de la tutelle est transmise au greffe du tribunal de grande instance dans le ressort duquel est née la personne protégée dans le but d'en porter l'existence sur le répertoire civil («RC») et de publicité par émargement de l'acte de naissance.

62. Voir, sur le calcul des degrés de parenté, l'article 743 du Code civil.

63. Cass., $1^{\text {re }}$ civ., 10 juillet 1984, no 83-10.653, Dalloz, 1984, Jur., p. 547, note J. Massip.

64. H. de Richemont, Sénat, 7 février 2007, p. 130. 
5 mars 2007 ne permettait pas de conclure que le législateur avait voulu briser la jurisprudence en cours sous l'empire de la loi du 3 janvier 1968, il était délicat de ne pas être attentif à la formulation de l'article 431 du Code civil qui sanctionne dorénavant l'absence de certificat médical circonstancié par l'irrecevabilité de la requête. Une sanction que la Cour de cassation impose aux juges du fond en annulant leurs jugements d'ouverture fondés sur un simple procès-verbal de carence ${ }^{65}$. Encouragés par la doctrine ${ }^{66}$, les juges du fond résistent; ils acceptent d'être régulièrement désignés par une requête même si le médecin choisi sur la liste du procureur de la République n'a pas pu rencontrer l'intéressé, pourvu que ce médecin rédige un certificat médical très circonstancié. Ce qui importe, précise un arrêt ${ }^{67}$ dans un obiter dictum, c'est que ce médecin habilité ait pu constater par lui-même le refus obstiné de l'intéressé de se laisser examiner alors qu'il était invité à se trouver à son domicile, à telle heure, par lettre recommandée avec accusé réception, et qu'il n'a pas voulu, dans ces conditions, ouvrir sa porte au médecin habilité qui annonçait sa venue. Ce médecin habilité, auquel ne peut être opposé le secret médical par ses médecins traitants ${ }^{68}$, doit ensuite caractériser au vu des éléments du dossier médical, l'altération des facultés mentales du patient et son besoin d'être assisté ou représenté dans les actes importants de la vie civile ${ }^{69}$. Quel que soit le contenu du certificat médical circonstancié, le secret médical doit être opposé au requérant. C'est pourquoi seul le procureur de la République et le juge des tutelles sont les destinataires de ce certificat médical ${ }^{70}$. Muni de ce précieux sésame, le requérant sera donc peut-être désavoué par l'intéressé qui lui préférera un autre parent que celui qui a eu l'audace de saisir le juge des tutelles.

\section{Le fonctionnement de la mesure}

Régulièrement saisi, le juge des tutelles entendra l'intéressé, le requérant et l'entourage familial, à moins que le médecin habilité n'ait indiqué un avis de non-audition dans le certificat médical circonstancié que le juge est libre néanmoins de ne pas suivre ${ }^{71}$. Il en va du respect des libertés individuelles de la personne à protéger. Lorsqu'elle est présente à l'audition, celle-ci indiquera les membres de sa famille ou de son entourage extrafamilial pour lesquels elle éprouve affection et respect et en qui elle place toute sa confiance. Le juge doit écouter cette parole et prendre en considération de tels sentiments ${ }^{72}$; il n'est pas tenu d'y obéir mais devra justifier dans son jugement les raisons pour lesquelles il a choisi d'investir tel parent plutôt que tel autre. L'article 449 du Code civil accorde sa préférence aux liens de couple, dès lors qu'il n'y a pas cessation de la vie commune entre les concubins, les partenaires pacsés ou les époux. Le basculement du droit matrimonial dans le droit tutélaire n'est pas neutre car le juge des tutelles est dorénavant investi, même à la demande du conjoint, de l'intérêt de l'époux à protéger ${ }^{73}$. La loi du 5 mars 2007 n'a pas repris la disposition selon laquelle l'époux est «de droit " ${ }^{74}$ curateur ou tuteur de son conjoint. Le juge est donc libre d'écarter le conjoint, comme le concubin. L'alinéa 2 de ce texte invite le juge à désigner un parent ou un allié pour assumer la fonction de curateur ou de tuteur.

Le juge détermine la nature de la mesure en fonction du besoin de la personne à protéger et désignera les organes de la protection. Il peut écarter la constitution d'un conseil de famille, même si cet organe est en principe nécessaire pour autoriser les actes de dispositions portant sur un bien d'une valeur supérieure à 50000 euros ${ }^{75}$, ce qui est souvent le cas lorsque le logement du majeur protégé est mis en

65. Cass., $1^{\text {re }}$ civ., 29 juin 2011, nº 10-21.879, Dalloz, 2011, Panor., p. 2502, obs. J.-M. Plazy; La semaine juridique, éd. G, 2011, 987, note N. Peterka; L'actualité juridique. Famille, 2011, p. 431, obs. T. Verheyde; Droit de la famille, 2011, comm. nº 133, note I. Maria; L'essentiel droit de la famille et des personnes, septembre 2011, p. 6, obs. G. Raoul-Cormeil; Revue trimestrielle de droit civil, 2011, p. 511, obs. J. Hauser, cassant le jugement d'appel du TGI Mont de Marsan, 8 octobre 2009; Dalloz, 2010, Jur., p. 2052, note T. Verheyde.

66. Aux observations citées supra (n. 65), adde N. Peterka, A. Caron-Déglise, F. Arbellot, Droit des tutelles: protection judiciaire et juridique des mineurs et des majeurs, $3^{\mathrm{e}}$ éd., Paris, Dalloz (Dalloz référence), 2012, $\mathrm{n}^{\circ} 43^{-25}$.

67. CA Douai, 11 janvier 2013, RG 12/05941, L'actualité juridique. Famille, 2013, p. 136, obs. G. Raoul-Cormeil («Notion de certificat de carence offrant des garanties similaires à un certificat médical circonstancié, une pièce procédurale essentielle à l'ouverture de la mesure de protection »). Rappr. CA Paris, 8 janvier 2013, pôle 3, ch. 7, RG 11/18841; Droit de la famille, mars 2013, comm. n 45, note I Maria; Revue trimestrielle de droit civil, 2013, p. 348, obs. J. Hauser.

68. Code civil, art. 431-1.

69. Code de procédure civile, art. 1219, al. $1^{\mathrm{er}}$.

70. Code de procédure civile, art. 1219, al. 3 .

71. Code civil, art. 432, al. $1^{\text {er }}$ : «Le juge statue, la personne entendue ou appelée». Voir aussi l'alinéa 2 de ce texte.

72. Code civil, art. 449 , al. 3 .

73. Pour une recherche du critère de cette combinaison, voir G. Raoul-Cormeil, «Le conjoint de la personne vulnérable (l'articulation du système matrimonial et du système tutélaire) », Defrénois, 2008, art. 38791, p. 1303-1319. Voir aussi M. Culioli, «La maladie d’un époux. Idéalisme et réalisme en droit matrimonial français", Revue trimestrielle de droit civil, 1968, p. 253-285; D. Langé, "Le conjoint de l'aliéné», Revue trimestrielle de droit civil, 1984, p. 33-68; V. Larribau-Terneyre, «Le régime matrimonial de l'incapable», La semaine juridique, éd. N, 1999, p. 843-849; J.-F. Sagaut, "Empêchement ou impéritie des époux: les solutions du droit des régimes matrimoniaux", L'actualité juridique. Famille, 2003, p. 124-129; C. Philippe, «Régimes matrimoniaux et altérations des facultés mentales», Droit de la famille, mai 2006, étude n²4, p. 33-36; N. Peterka, "Les dispositifs alternatifs de protection de la personne mariée», L'actualité juridique. Famille, 2012, p. 253.

74. Code civil, anc. art. 496 et 509-1 (loi no 68-5 du 3 janvier 1968), réciproquement en tutelle et en curatelle. Rappr. Code Napoléon (21 mars 1804), anc. art. 506: «Le mari est, de droit, le tuteur de sa femme interdite». Voir aussi anc. art. 507: «La femme pourra être nommée tutrice de son mari. En ce cas, le conseil de famille réglera la forme et les conditions de l'administration [...]».

75. Décret $\mathrm{n}^{\circ}$ 2008-1484 du 22 décembre 2008, art. 4, al. $1^{\text {er }}$. 
vente. Le juge peut aussi canaliser les tensions familiales et investir plusieurs parents de fonctions concurrentes afin de rassurer la famille. La division de la curatelle ou de la tutelle entre la protection de la personne et des biens permet de prendre en compte les différentes compétences des membres de la famille et la proximité des liens entre les parents. La désignation d'un subrogé curateur ou d'un subrogé tuteur permet également de trouver un remède commode et efficace en cas d'opposition d'intérêts et d'investir un autre parent d'une prérogative de contrôle et d'alerte. À l'inverse, le juge des tutelles peut abréger les contraintes, en dispensant le tuteur, par exemple, de l'obligation de dresser un inventaire avec deux témoins majeurs extérieurs à la famille ${ }^{76}$. L'article 512 du Code civil permet aussi au juge des tutelles de dispenser le tuteur, lorsque la tutelle n'a pas été confiée à un mandataire judiciaire à la protection des majeurs et que les revenus et le patrimoine de la personne protégée sont modiques, de l'obligation d'établir le compte annuel de gestion. C'est en pratique ce que décident les juges des tutelles lorsque les relations familiales sont apaisées, une telle décision présentant l'avantage de décharger le greffe du contrôle des comptes de gestion ${ }^{77}$. Si la protection des personnes vulnérables est un devoir des familles, il faut préciser qu'elles doivent s'acquitter gratuitement de cette charge ${ }^{78}$, sans pouvoir y renoncer au-delà de cinq ans lorsqu'elle est confiée à l'autre membre du couple ou à un enfant ${ }^{79}$. L'allégement des contraintes ne doit pas inquiéter la famille car le tuteur répond des fautes commises dans sa gestion dans les cinq ans suivant la cessation de la mesure ${ }^{80}$. Pour la curatelle, la loi a rehaussé le seuil de la faute au dol ou à la faute grave ${ }^{81}$, sans suspendre le cours de la prescription extinctive ${ }^{82}$. Le juge des tutelles peut à l'inverse multiplier les précautions contre les détournements des revenus du majeur protégé, la plus grande des précautions étant d'écarter la famille en investissant un professionnel.

\section{Les familles écartées de la protection des majeurs vulnérables}

Le juge des tutelles doit indiquer les raisons pour lesquelles il investit un mandataire judiciaire à la protection des majeurs de la fonction de curateur ou de tuteur (A). Un tel recours n'est pas neutre sur le coût de la mesure mais également sur les prérogatives que la loi lui donne dans l'exercice des droits familiaux de la personne protégée (B).

\section{A. Le recours au corps des mandataires judiciaires à la protection des majeurs (MJPM)}

La désignation d'un mandataire judiciaire à la protection des majeurs se justifie par des causes diverses. Les fondements de l'exclusion de la famille (1) trouvent leur garantie dans le statut professionnel des mandataires judiciaires à la protection des majeurs (2).

\section{Les fondements de l'exclusion de la famille}

Si longue et si détaillée, la loi du 5 mars 2007 fait preuve d'une étonnante sobriété à l'article 450 du Code civil :

Lorsqu'aucun membre de la famille ou aucun proche ne peut assumer la curatelle ou la tutelle, le juge désigne un mandataire judiciaire à la protection des majeurs inscrit sur la liste prévue à l'article L. 471-2 du code de l'action sociale et des familles.

Sans ambages mais avec fermeté, la loi reste fidèle au principe de préférence familiale ${ }^{83}$, un principe présent dans les travaux préparatoires ${ }^{84}$ de la loi du 3 janvier 1968 et que la Cour de cassation a su formuler avec plus de clarté:

76. Code de procédure civile, art. 1253.

77. M.-P. Régnault-Lugbull, «La protection familiale d'une personne majeure et le juge des tutelles», in Nouveau droit des majeurs protégés..., p. 269 sq., spéc. p. 278

78. Code civil, art. 419, al. $1^{\text {er }}$, qui permet cependant au juge des tutelles de fixer le montant des indemnités que le majeur protégé devra payer à son tuteur, curateur ou mandataire spécial, s'il est chargé d'une mission de gestion assez lourde eu égard aux biens en cause.

79. Code civil, art. 453 .

80. Code civil, art. 515 .

81. Code civil, art. 421 in fine.

82. Code civil, art. 2235, interprété a contrario.

83. Voir, en ce sens, la circulaire de la Direction des affaires civiles et du Sceau n ${ }^{\circ}$ CIV/o1/o9/C1 du 9 février 2009 (BOMJ, n 2009 -01 du 28 février 2009, publié le 2 avril suivant), qui consacre deux pages (p. 30-31) à «La priorité aux liens familiaux, d'affection et de confiance» avant de porter l'analyse sur les mandataires judiciaires à la protection des majeurs. La doctrine a livré une interprétation similaire des textes à propos de la qualité du mandataire judiciaire: J. Hauser, «La famille et l'incapable majeur», L'actualité juridique. Famille, 2007, p. 198; G. Kessler, V. Zalewski, «Le principe de primauté de la famille après la réforme des incapacités par la loi du 5 mars 2007 ", Revue Lamy droit civil, n 41 , septembre 2007 , étude $n^{\circ} 2665$, p. 34; N. Peterka, «La famille dans la réforme...", passim; A. Batteur, "Majeurs protégés. Dispositions communes à toutes les protections. Articles 415 à 432 ", Jurisclasseur Code civil, fascicule no 12 , janvier 2009, nº 10, p. 5, où l'auteur souligne que «le traditionnel équilibre entre le rôle de la famille et le rôle de l'État (par l'intermédiaire du juge des tutelles) s'était avéré fragile. Il paraissait indispensable [au législateur de 2007] de renforcer le recours aux familles».

84. J. Carbonnier, Droit civil, t. 1, Introduction, les personnes, l’enfant, le couple, Paris, PUF (Quadrige. Manuels), 2004, n³34, spéc. p. 653: «Des raisons scientifiques conduiraient à la tenir à distance, la thèse étant que la maladie de l'individu est la manifestation d'un trouble psychique latent dans le milieu familial. Mais, en sens opposé, on ne saurait méconnaître que les mesures de protection appliquées au malade atteignent gravement ses proches, désorganisent son foyer, déstabilisent son conjoint et ses enfants. Écarter les parents, c'est priver les médecins et les juges d'une information utile sur l'origine et le parcours du trouble mental, c'est aussi priver le malade d'un soutien affectif. Joint que la présence de 
Si l'esprit général de la loi implique que préférence soit donnée, chaque fois qu'il est possible, à la tutelle familiale, il appartient au juge des tutelles et, sur recours, au tribunal de grande instance de déterminer souverainement quel est, eu égard notamment à l'intérêt de l'incapable, le mode d'exercice de la tutelle qui est le plus approprié ${ }^{85}$.

L'intérêt du majeur protégé est sans aucun doute le cap que ne doit pas perdre de vue le juge mais la loi ne nous dit pas quels obstacles le juge doit éviter lorsqu'il qui tient le gouvernail. L'emploi du verbe "pouvoir» est assez vague. Fait-il référence à toute sorte d'inaptitude de la famille? Des empêchements? Ou faut-il prendre en compte le refus car ne pas vouloir assumer la charge curatélaire ou tutélaire c'est aussi ne pas se donner les moyens d'honorer le mandat judiciaire? La désignation d'un gérant de tutelle et, depuis le $1^{\mathrm{er}}$ janvier 2009, d'un mandataire judiciaire à la protection des majeurs, suscite le mécontentement des familles et certaines d'entre elles n'hésitent pas à porter leur recours aussi loin que le leur permet le Code de la procédure civile. La systématisation d'une douzaine d'arrêts, souvent non publiés au Bulletin, permet de préciser le contenu du critère d'exclusion de la famille. D'abord, il convient de préciser que les juges du fond bénéficient d'un pouvoir souverain d'appréciation pour choisir la personne qui sera la plus compétente pour exercer la mesure ${ }^{86}$. Ils doivent cependant indiquer les motifs en vertu desquels ils justifient l'exclusion de la famille du majeur protégé, sans quoi ils s'exposent à une cassation pour manque de base légale ${ }^{87}$. La Cour de cassation exerce ici un contrôle allégé sur la logique de la motivation et leur rappelle que les juges doivent prendre en considération les sentiments exprimés par le majeur protégé, comme le leur ordonne déjà l'article 449, alinéa 3 du Code civil. Ensuite, les juges doivent être attentifs aux difficultés dans lesquelles sont placés les membres de la famille. Ainsi l'épouse souffrant d'un important handicap physique n'a pas été jugée apte à assister dans les actes importants de la vie courante son mari qu'un grave accident de la circulation a laissé handicapé et fragile à cause de troubles de la personnalité ${ }^{88}$. Les difficultés peuvent également être d'ordre social. La protection de la personne et des biens d'un majeur protégé suppose l'aptitude à solliciter de l'administration des prestations sociales, ce à quoi ne parviendront pas les membres de la famille qui ne maîtrisent pas la langue française $^{89}$. Par ailleurs, l'inaptitude ${ }^{90}$ à gérer les biens du majeur protégé est illustrée par le fait que le tuteur familial produit des comptes de gestion «opaques et illisibles ${ }^{91}$. La décision d'écarter ce membre de la famille est d'autant plus fondée que la personne investie de la fonction de tuteur ou de curateur délaisse le majeur protégé. Le mal à prévenir n'est pas moins grand lorsque, par son attitude, la mère du majeur protégé « déstabilise les équipes soignantes, perturbe son fils et compromet son projet de soins ${ }^{92}$. À cet état psychologique de fusion, s'ajoutent toutes les situations de conflits familiaux ${ }^{93}$. La contrariété d'intérêts connaît de nombreuses illustrations parmi celle où un procès en annulation du mariage éloigne les époux et disqualifie le conjoint du majeur protégé à prendre en charge la personne et les biens de son époux ${ }^{94}$. C'est à cette catégorie de contrariété d'intérêts qu'il faudrait rattacher la situation du majeur protégé qui est un bourreau pour ses enfants mineurs comme pour son époux ou sa concubine à laquelle il manque de respect chaque jour. Loin d'être une hypothèse d'école, cette situation justifie de protéger la famille du majeur protégé contre lui-même. Le juge des tutelles, gardien du seul intérêt du majeur protégé, saisit le procureur de la République de ces difficultés dès qu'il en est informé. Dans tous les cas, la nomination d'un tiers impartial offre des garanties suffisantes que la loi du 5 mars 2007 a renforcées en forgeant un statut à ces professionnels qu'elle nomme mandataire judiciaire à la protection des majeurs.

la famille est un contrepoids salutaire à l'intervention de l'autorité, judiciaire ou médicale, dans la vie privée. Balance faite de ces arguments contradictoires, le principe s'est affirmé d'une primauté des organes familiaux dans les trois régimes de protection ».

85. Cass., $1^{\text {re }}$ civ., 17 mars 1992, $\mathrm{n}^{\circ}$ 90-10.892, Bulletin civil I, $\mathrm{n}^{\circ} 88$; Dalloz, 1993, Jur., p. 17 ( $1^{\mathrm{re}}$ espèce), note J. Massip; Revue trimestrielle de droit civil, 1992, p. 541, obs. J. Hauser.

86. Cass., $1^{\text {re }}$ civ., 29 février 1984, $\mathrm{n}^{\circ} 83-10.092$, Bulletin civil $I, \mathrm{n}^{\circ} 83$; Dalloz, 1984, Jur., p. 423, note J. Massip.

87. Cass., $1^{\text {re }}$ civ., 10 octobre 1984, Bulletin civil I, $\mathrm{n}^{\circ} 254$; Dalloz, 1985, Jur., p. 61, note J. Massip; Rapport de la Cour de cassation 1985, Paris, La documentation française, p. 36. À partir de cet arrêt et jusqu'au $1^{\text {er }}$ janvier 2009, la Cour de cassation exigeait des juges du fond qui désignaient un gérant de tutelles de se justifier au regard de la situation familiale et patrimoniale de l'intéressé, car si les biens étaient importants, alors une tutelle complète s'imposait en lieu et place du gérant de tutelles. Voir aussi Cass., $1^{\text {re }}$ civ., 5 décembre 2012, $\mathrm{n}^{\circ}$ 11-26.611, La semaine juridique, éd. G, 2013, 104, note N. Peterka; L'essentiel droit de la famille et des personnes, janvier 2013, p. 5, obs. G. Raoul-Cormeil. Voir toutefois, pour une cassation pour violation de la loi: Cass., $1^{\text {re }}$ civ., $1^{\text {er }}$ décembre 2010, $\mathrm{n}^{\circ}$ 09-16.764, Droit de la famille, 2011, comm. $n^{\circ} 23$, note I. Maria.

88. Cass., $1^{\text {re }}$ civ., 25 novembre 2009, $\mathrm{n}^{\circ}$ 08-25.492, Les petites affiches, 10 mars 2010, p. 4 ( $2^{\mathrm{e}}$ espèce), note G. Raoul-Cormeil.

89. Cass., $1^{\text {re }}$ civ., 8 avril $2009, \mathrm{n}^{\circ} 08-11.417$.

90. Voir le cas où les juges du fond avaient constaté l'inaptitude du fils de la personne en tutelle à gérer ses biens; Cass., $1^{\text {re }}$ civ., 9 janvier 1996 , n 93-18.704, Defrénois, 1996, p. 1004, obs. J. Massip.

91. Cass., $1^{\text {re }}$ civ., 9 décembre 2009, $n^{\circ}$ 08-14.023, Les petites affiches, 10 mars 2010, p. 4 ( $4^{\mathrm{e}}$ espèce).

92. Cass., $\mathrm{1}^{\mathrm{re}}$ civ., 9 décembre $2009, \mathrm{n}^{\circ}$ 07-13.981, Droit de la famille, février 2010, comm. $\mathrm{n}^{\circ} 50$, note I. Maria; L'essentiel du droit des personnes et de la famille, février 2010, p. 3, obs. G. Raoul-Cormeil; Les petites affiches, 10 mars 2010, p. 4 ( $3^{\mathrm{e}}$ espèce).

93. Cass., $1^{\mathrm{re}}$ civ., 25 novembre 2012, $\mathrm{n}^{\mathrm{o}} 08-20.271$, Les petites affiches, 10 mars 2010, p. 4 ( $1^{\mathrm{re}}$ espèce).

94. Voir notamment Cass., $1^{\text {re }}$ civ., 20 décembre 2000, $\mathrm{n}^{\circ}$ 99-10.474, Les petites affiches, 11 juin 2001, note J. Massip; Cass., $1^{\text {re }}$ civ., 23 février 2011 , $\mathrm{n}^{\circ}$ 10-12.923 et n⿳0 10-12.924, Dalloz, 2011, Panor., p. 2515, obs. J.-J. Lemouland; La semaine juridique éd. N, 2011, 1244, étude J. Massip.; Cass., $1^{\text {re }}$ civ., 12 septembre 2012, $\mathrm{n}^{\circ}$ 11-17.246, Droit de la famille, novembre 2012, comm. $\mathrm{n}^{\circ}$ 172, note I. Maria; L'essentiel droit de la famille et des personnes, octobre 2010, n 159, p. 5, obs. G. Raoul-Cormeil; Revue trimestrielle de droit civil, 2012, p. 712, obs. J. Hauser. 


\section{Le statut de MJPM}

Le mandataire judiciaire à la protection des majeurs est un auxiliaire de justice ${ }^{95}$ qui exerce, au nom de la collectivité publique ${ }^{96}$, les mesures de protection juridique lorsqu'elles ne peuvent être confiées à la famille. La loi du 5 mars 2007 a maintenu les trois modes d'exercice de cette profession. Les préfets de département agréent des personnes physiques, qu'il s'agisse des gérants privés qui exercent en leur nom personnel cette activité libérale ou des préposés des établissements publics de santé qui hébergent, parmi leurs patients, des majeurs protégés. Les préfets de département délivrent aussi des autorisations à des associations tutélaires. Plus de $93 \%$ des mesures sont confiées à ces personnes morales. Ces professionnels ont en commun d'être rémunérés pour l'accomplissement de cette charge dans des conditions fixées par décret ${ }^{97}$. Sans être encore une profession réglementée ${ }^{98}$, exerçant sous l'égide d'un ordre professionnel et d'un code de déontologie, les mandataires judiciaires à la protection des majeurs n'ont pas un statut unifié. Les mandataires libéraux et les associations tutélaires doivent justifier d'une assurance professionnelle alors que les préposés d'établissement public exercent ce service public ${ }^{99}$ dans le cadre de l'administration où l'État est son propre assureur. De surcroît, comme les préposés d'établissements publics de santé, les salariés des associations tutélaires bénéficient de l'immunité du salarié qui ne cède qu'en cas d'infraction pénale intentionnelle ${ }^{100}$. Ainsi brossé à grand traits ${ }^{101}$, le statut du mandataire judiciaire à la protection des majeurs offre des garanties au juge qui écarte un membre de la famille au profit d'un tiers impartial et désintéressé, ce qui ne va pas sans poser de difficulté lorsqu'il doit s'immiscer dans la vie intime du majeur protégé pour l'exercice de ses droits familiaux.

\section{B. Le rôle des MJPM dans l'exercice des droits familiaux}

Le rôle du mandataire judiciaire à la protection des majeurs est plus ou moins invasif selon qu'il s'agit de (dé)nouer une relation filiale ou parentale ou de (dé)lier une relation de couple.

\section{L'enfant}

L'article 458 du Code civil indiquant, à l'alinéa 2, à titre d'illustration, «la déclaration de naissance d'un enfant et sa reconnaissance, [...] le consentement donné à sa propre adoption ou à celle de son enfant», on pourrait bien se demander si tous les actes juridiques relatifs à l'établissement de la filiation n'appartiennent pas à la catégorie des droits strictement personnels que le majeur protégé doit accomplir seul, sans possibilité d'être assisté ni représenté. Critiqué pour des raisons de technique juridique ${ }^{102}$ ou de légistique ${ }^{103}$, ce texte ne dit cependant pas que le mandataire judiciaire à la protection des majeurs ne doit rien faire. S'il n'a pas de consentement à donner, ce professionnel peut néanmoins accompagner le majeur protégé pour s'assurer que sa parole sera prise au sérieux et entendue par l'officier de l'état civil. Il y aurait beaucoup à dire sur cette notion d'accompagnement qui a fait son entrée par le Code de la santé publique, à propos de l'interruption volontaire de grossesse décidée par une personne mineure ${ }^{104}$, et qui trouve dans le nouveau droit des majeurs protégés des applications aussi nombreuses ${ }^{105}$ qu'inédites qu'il plaira aux rédacteurs du Code de déontologie des mandataires judiciaires à la protection des majeurs de développer. Quoi qu'il en soit, ne serait-il pas hasardeux de considérer

95. Voir l'étude de L. Raschel, «Le mandataire judiciaire à la protection des majeurs est-il un auxiliaire de justice? », Droit de la famille, décembre 2012, étude $\mathrm{n}^{\circ} 14$, p. 21-24.

96. Voir spécialement G. Lebreton, «L'exclusion de la famille dans la protection des majeurs sous l'angle du droit public», in Nouveau droit des majeurs protégés..., p. 259-267. Cette manifestation doit être replacée dans un contexte plus général qu'un auteur a étudié avec une belle hauteur de vue: J. Hauser, «Une famille récupérée», in Le droit privé français à la fin du XX siècle: études offertes à Pierre Catala, Paris, Litec, $2001, \mathrm{p} .327$.

97. Voir le décret nº 2008-1554 du 31 décembre 2008 relatif aux modalités de participation des personnes protégées au financement de leur mesure de protection.

98. Voir l'étude de M. Couturier, «Le mandataire judiciaire à la protection des majeurs exerce-t-il une profession réglementée?», Droit de la famille, décembre 2012, étude $n^{\circ} 16$, p. 27-30.

99. Voir l'étude de S. Guérard, «Les missions du mandataire judiciaire à la protection des majeurs relèvent-elles d'un service public?», Droit de la famille, décembre 2012, étude $\mathrm{n}^{\circ} 15, \mathrm{p} .24-27$.

100. Voir spécialement A. Dumery, «Responsabilité civile des organes de protection: la nouvelle donne», Revue de droit sanitaire et social, 2008 , $\mathrm{n}^{\circ}$ 5, p. 844-850; M. Rebourg, "La responsabilité civile des mandataires judiciaires à la protection des majeurs dans l'exercice de leurs missions", Droit de la famille, juillet-août 2010, étude n 17, p. 12-17; E. Frago, "Le délégué à la protection des majeurs», in Nouveau droit des majeurs protégés..., p. 63-78, spéc. p. 73 sq.

101. Voir spécialement N. Peterka, A. Caron-Déglise, F. Arbellot, Droit des tutelles..., $\mathrm{n}^{\circ}$ 46, p. 242-275; Curatelle, tutelle, accompagnements..., $\mathrm{n}^{\circ}$ 489, p. 217; J. Massip, Tutelle des mineurs et protection juridique des majeurs, Paris, Defrénois-Lextenso, 2009, nº 417 sq., p. 343-350. Voir aussi G. RaoulCormeil, "Atouts et faiblesses du statut professionnel de mandataire judiciaire à la protection des majeurs», Droit de la famille, décembre 2012, étude no 13, p. 16-21; A. Batteur, "Synthèse et perspectives de la profession de MJPM», Droit de la famille, décembre 2012, étude n 17, p. 31-34.

102. Voir A. Batteur, "Le majeur protégé et l'enfant», in Nouveau droit des majeurs protégés..., p. 223-242, spéc. p. 233, qui reproche au texte de confondre l'indication de l'acte de naissance qui doit être faite dans les trois jours ouvrés de la naissance selon les déclarations faites par les témoins de l'accouchement (Code civil, art. 56), avec l'indication du nom de la mère, voire du mari de la mère, qui établit la filiation maternelle (Code civil, art. 311-25) et paternelle (Code civil, art. 312).

103. Voir spécialement P. Salvage-Gerest, «Les actes dont la nature implique le consentement strictement personnel en tutelle: une catégorie à revoir d'urgence», Droit de la famille, mars 2009, étude n 17, p. 19-24, où l'auteur dénonce les effets pervers de ce texte.

104. Code de la santé publique, art. L. 2212-4, al. 2 in fine. Voir aussi, dans le même Code, art. L. 1111-5, al. $1^{\text {er }}$ in fine: «Dans ce cas, le mineur se fait accompagner d'une personne majeure de son choix».

105. Par-delà les mesures d'accompagnement social personnalisé et mesures d'accompagnement judiciaires. Sur lesquelles, voir L. Mauger-Vielpeau, «Le retour du prodigue», La semaine juridique, éd. N, 2008, étude nº 1269, p. 27-32. 
que le majeur protégé pourrait engager seul une action relative à la filiation devant le tribunal de grande instance sans avoir à solliciter la personne chargée de la mesure de protection? Il peut déjà paraître délicat pour un majeur protégé de manifester la volonté de reconnaître un enfant devant un officier de l'état civil, mais il est bien plus difficile d'engager une action relative à la filiation car il faudra solliciter le ministère d'un avocat et conclure un mandat ad litem. Confrontée à des difficultés pratiques si éloignées des idéaux poursuivis par le législateur, la doctrine n'a guère envisagé ces situations dans les premières années d'application de la loi nouvelle ${ }^{106}$. Mais si faible soit le contentieux de la filiation si on le compare aux nombres de naissance et d'accouchement sous X qui mettent en cause des personnes vulnérables, faut-il pour autant considérer que les actions relatives à la filiation appartiennent à la catégorie des droits strictement personnels. À l'argument de texte ${ }^{107}$, l'article 458 du Code civil étant interprété par analogie, un grand auteur ajoute que la distinction entre les droits patrimoniaux et extrapatrimoniaux est assez «peu opérationnelle» ${ }^{108}$, car

[...] la plupart des actions d'état en réclamation ou en contestation sont inspirées, au moins pour une grande partie, par des préoccupations patrimoniales, comme [...] venir à la succession ou exclure le droit de succéder ${ }^{109}$.

Si l'enjeu de cette qualification est à peu près nul en matière de curatelle, où le curatélaire doit toujours être assisté de son curateur lorsqu'il engage une action en justice ou y défend ${ }^{110}$, il n'en est pas de même en matière de tutelle, où le tuteur ne peut engager d'action extrapatrimoniale sans saisir le juge des tutelles ${ }^{111}$. Face à une telle classification, la question est seulement de savoir s'il est raisonnable de laisser au seul majeur protégé le soin de prendre la décision d'engager une action relative à la filiation ou si cette décision ne doit pas plutôt être prise par le curatélaire assisté de son curateur ${ }^{112}$ ou par le juge des tutelles alors saisi par le tuteur du majeur protégé. Le problème ainsi posé, il semble raisonnable de ne pas trop étendre le domaine d'application de l'article 458 du Code civil et d'élever une limite au règne discret de l'analogie. D'ailleurs à bien y regarder, l'alinéa 2 ne vise que le consentement donné par le majeur protégé à sa propre adoption ou à celle de son enfant. La situation de la demande en adoption n'est pas tout à fait identique car le candidat à l'adoption doit être à même de manifester un projet parental, de solliciter un agrément auprès des services d'un conseil général puis de former une requête devant le tribunal de grande instance. Au regard de ce processus, bien plus lourd que la question du consentement à l'adoption, il ne serait pas déraisonnable de sortir l'acte d'adoption de la catégorie des droits strictement personnels. La proposition d'une telle qualification ne se justifie pas dans le but d'élever un filtre à une manifestation de volonté qui pourrait sembler capricieuse ou farfelue, en espérant que le curateur ou le juge des tutelles se saisisse de leur prérogative pour élever un veto à une demande formulée à la légère, même si cette précaution n'est pas complètement à négliger. Cette proposition prend corps dans les difficultés pratiques de mise en œuvre des droits personnels du majeur protégé que le curateur ou le tuteur doit être à même de mettre en œuvre sérieusement dans l'intérêt du majeur protégé. En attendant la jurisprudence qui balaiera ces incertitudes, passons au rôle du mandataire judiciaire à la protection des majeurs en matière de vie de couple.

\section{Le couple}

Si l'on excepte le concubinage qui, parce qu'il est un fait juridique, n'appelle guère de réponse du droit des majeurs protégés si ce n'est la difficulté dans laquelle se mettrait la personne du majeur protégé qui s'installerait avec un être qui abuserait de sa confiance et le soumettrait à un traitement malveillant ${ }^{113}$, le mariage et le pacs, comme le divorce et la dissolution du pacs ne sont pas des décisions ${ }^{114}$ que peut prendre seul le curatélaire ou le tutélaire sans en parler avec le mandataire judiciaire à la protection des majeurs. Le rôle joué par le curateur ou le tuteur varie selon qu'il doit être saisi avant la décision de s'unir ou, par contraste, en cas de désunion, selon qu'il doit être saisi après coup pour mettre en œuvre la décision de la rupture. Développons. Ainsi, pour le mariage, le curatélaire comme le tutélaire doit être autorisé par une tierce personne avant de se marier. Le curateur est investi de la prérogative d'autoriser le mariage du curatélaire, alors que le tuteur devra saisir le juge des tutelles. Dans les deux cas, cette décision est une sage précaution dans la mise en œuvre de la liberté nuptiale car le mariage est riche de nombreux effets juridiques à l'égard de la personne comme

106. Voir spécialement J. Hauser, «Le majeur protégé, acteur familial», Droit de la famille, février 2011, étude n 6, p. 28-33; J. Roche Dahan, «L’accès du majeur protégé à la procréation et à la filiation», Revue Lamy droit civil, n 83, juin 2011, étude no 4290, p. 80-87; A. Batteur, «Le majeur protégé et l'enfant».

107. J. Hauser, "La distinction des actions personnelles et des actions patrimoniales à l'épreuve du droit des majeurs protégés», in Mélanges en l'honneur du professeur Gérard Champenois, p. 441-451, spéc. p. 448, texte et note 27.

108. Ibid., p. 447.

109. Ibid.

110. Code civil, art. 468 , al. 3 .

111. Code civil, art. 475 , al. 2 .

112. Que la curatelle soit simple ou renforcée.

113. Voir notamment T. Verheyde, «Le juge des tutelles, nouveau juge aux affaires familiales?», Dalloz, 2010, Chron., p. $2460-2464$.

114. Sur lesquelles, voir J. Hauser, «Le majeur protégé, acteur familial»; I. Barrière-Brousse, «La vie de couple du majeur protégé», Revue Lamy droit civil, n 83, juin 2011, étude n 4289, p. 74-79; L. Mauger-Vielpeau, «Le couple et le majeur protégé», in Nouveau droit des majeurs protégés... p. $243-258$. 
des biens du majeur protégé: secours, fidélité, assistance ${ }^{115}$, communauté de vie ${ }^{116}$, solidarité des dettes ménagères ${ }^{117}$ et la communauté réduite aux acquêts qui s'impose aux époux pour le cas où ils n'auraient pas conclu de contrat de mariage ${ }^{118}$. Aux curateurs et aux tuteurs d'être vigilants et de solliciter d'un notaire une analyse patrimoniale de la situation du majeur protégé pour déterminer si le régime légal lui convient ou si un régime conventionnel ne lui conviendrait pas mieux. L'intérêt du majeur protégé peut être diversement apprécié, la Cour de cassation laissant aux juges du fond un pouvoir souverain ${ }^{119}$. C'est pourquoi l'existence des recours est la seule garantie qui permette de s'assurer que l'autorisation à mariage n'est pas une entrave à l'exercice de la liberté nuptiale ${ }^{120}$. De même, le tutélaire devra solliciter l'autorisation du juge des tutelles ${ }^{121}$ pour être habilité à l'assister à la conclusion du pacs ${ }^{122}$. Le curateur assistera le curatélaire lors de la conclusion de ce pacte $^{123}$. En revanche, ni le curateur ${ }^{124}$, ni le tuteur ${ }^{125}$ n'ont de rôle à jouer lorsque les partenaires déclarent devant le greffier leur intention de vivre ensemble en pacs. La conclusion d'un pacs notarié intervertit l'étape de la déclaration et de la signature de l'acte authentique, ce qui oblige le curateur et le tuteur à être présents lors de la signature. La modification du pacs est soumise au même régime. En revanche, les précautions s'inversent pour la rupture. La décision de rompre le pacs appartient au seul majeur protégé, en curatelle ${ }^{126}$ comme en tutelle ${ }^{127}$, le curateur ${ }^{128}$ et le tuteur ${ }^{129}$ ayant un rôle à jouer dans la mise en œuvre de cette décision: la signification au partenaire répudié, la publicité sur les registres du greffier ou du notaire et l'émargement de l'acte de naissance, sans compter avec les opérations de liquidation patrimoniale. On retrouve en matière de divorce un régime analogue, à la différence près que le législateur interdit au curatélaire comme au tutélaire de divorcer par consentement mutuel ou pour acceptation du principe de la rupture du mariage ${ }^{130}$. Le curatélaire et le tutélaire ne peuvent pas, seuls, engager une procédure en divorce. Le tutélaire devra convaincre son tuteur de solliciter une autorisation du juge des tutelles ${ }^{131}$. Le curatélaire devra être assisté de son curateur ${ }^{132}$. Mais la loi permet aussi au tuteur, en tutelle seulement, de prendre l'initiative de la rupture. Le tuteur devra cependant convaincre le juge des tutelles que le tutélaire a plus d'inconvénients que d'avantages à rester marier. La consultation du médecin traitant ${ }^{133}$ a pour but de vérifier que la rupture de ce lien personnel n'est pas de nature à porter atteinte à sa santé en le plaçant dans une situation de dénuement ou de culpabilité. Le tuteur peut aussi prendre l'initiative de rompre unilatéralement le pacs: la saisine du juge des tutelles aux fins d'autorisation est aussi nécessaire ${ }^{134}$, sans qu'il soit ici besoin d'accompagner la requête d'une attestation médicale qui, pourtant, ne serait pas superflue. Mais, dans tous les cas, le juge des tutelles est obligé d'entendre le tutélaire. De telles prérogatives paraissent exorbitantes lorsqu'elles sont mises en œuvre par un mandataire judiciaire à la protection des majeurs dont la charge curatélaire ou tutélaire est, dans le silence du jugement, étendue à la protection de la personne ${ }^{135}$.

En définitive, il faut espérer que les familles prendront soin de leurs membres dès qu'ils deviendront vulnérables. La rencontre de la famille avec le juge des tutelles saisi par le procureur de la République au cours de l'audience précédant l'ouverture de la mesure de protection juridique sera peut-être le lieu d'une prise de conscience

115. Code civil, art. 212.

116. Code civil, art. 215.

117. Code civil, art. 220.

118. Code civil, art. 1400

119. Voir spécialement Cass., $1^{\mathrm{re}}$ civ., 5 décembre 2012, $\mathrm{n}^{\mathrm{o}} 11-25.158$, Droit de la famille, 2013, comm. $\mathrm{n}^{\mathrm{o}} 14$, note I. Maria; L'essentiel droit de la famille et des personnes, janvier 2012, $\mathrm{n}^{\circ} \mathrm{021}, \mathrm{p} .3$, obs. G. Raoul-Cormeil: «C'est à bon droit que la cour d'appel, après avoir retenu que $\mathrm{M}^{\mathrm{me}} \mathrm{X}$... s'était mariée dans le but exclusif d'appréhender le patrimoine de Philippe Y..., en a déduit, sans méconnaître les exigences conventionnelles de la liberté du mariage, qu'il y avait lieu d'annuler celui-ci, faute de consentement».

120. CC, déc. $\mathrm{n}^{\circ}$ 2012-26o QPC du 29 juin 2012, Droit de la famille, septembre 2012, comm. nº 136, note M. Bruggeman et octobre 2012, comm. ${ }^{\circ} 148$, note I. Maria; Revue trimestrielle de droit civil, 2012, p. 510, obs. J. Hauser; G. Raoul-Cormeil, «L'autorisation requise pour le mariage du curatélaire, sage précaution dans l'exercice de la liberté nuptiale», Dalloz, 2012, Point de vue, p. 1899. Prise en matière de curatelle, cette décision a une portée plus grande; elle s'impose à la tutelle par un raisonnement a fortiori. En outre, elle peut être transposée, à l'égard de ces deux mesures, pour la conclusion du pacs (Code civil, art. 461 et 462 ).

121. Code civil, art. 462 , al. $1^{\text {er }}$.

122. Code civil, art. 462, al. 2.

123. Code civil, art. 461 , al. $1^{\text {er }}$.

124. Ibid.

125. Code civil, art. 462, al. 2.

126. Code civil, art. 461 , al. 3

127. Code civil, art. 462, al. 4 (rupture unilatérale à l'initiative du tutélaire) et al. 6 (décision conjointe des partenaires pacsés, majeur(s) protégé(s) ou non).

128. Code civil, art. 461 , al. 3 et 4 .

129. Code civil, art. 462 , al. 4 et 7 .

130. Code civil, art. 249-4.

131. Code civil, art. 249, al. $\mathbf{1}^{\text {er }}$.

132. Code civil, art. 249, al. 2.

133. Code civil, art. 249 , al. $\mathbf{1}^{\text {er }}$.

134. Code civil, art. 462, al. 5 .

135. Code civil, art. 451, al. 2. 
tardive. Ainsi éclairé, le juge désignera la personne la plus compétente et la plus intègre pour protéger un majeur vulnérable contre lui-même et lui éviter qu'il ne soit victime de sa propre faiblesse. Parfois nécessaire, l'investiture d'un mandataire judiciaire à la protection des majeurs ne présente pas que des avantages. D’abord, cette forme de protection juridique présente un coût pour le majeur protégé ou pour la collectivité publique lorsque celui-ci est bénéficiaire de droits sociaux. Ensuite, les professionnels du dispositif tutélaire n'auront jamais la disponibilité que peut déployer une personne pour un membre de sa famille. L'intervention d'un mandataire judiciaire à la protection des majeurs est cependant la meilleure solution lorsque le majeur à protéger est en danger parmi les siens ou lorsqu'il se montre malveillant à l'égard des membres de sa propre famille. C'est là toute la richesse de la loi du 5 mars 2007 de comprendre un large éventail de dispositifs pour répondre aux vicissitudes de la vie familiale avec plus de précaution sans doute, mais avec la même tolérance que celle dont le législateur a fait preuve à l'égard des personnes jouissant de leur pleine capacité civile. 